\title{
A Case Study of Balance Rehabilitation in Parkinson's Disease
}

\author{
Stanley John Winser (Corresponding author) \\ Physiotherapy lecturer \\ Masterskill University College of Health Sciences (MUCH), Malaysia \\ Tel: 61-4927-1374Ｅ-mail: stanley@masterskill.edu.my \\ Priya Kannan \\ Physiotherapy lecturer \\ Masterskill University College of Health Sciences (MUCH), Malaysia
}

Received: September 19, $2010 \quad$ Accepted: October 12, 2010 doi:10.5539/gjhs.v3n1p90

\begin{abstract}
There is evidence to say that balance can be improved by manipulating peripheral sensory inputs. Our hypothesis was, improvement in the inputs from proprioceptors using sensory specific balance training would improve balance. We intend to document the influence of training proprioceptors in improving balance among Parkinsonism. Single case study of a 65 years old parkinson's subject was considered. Trial was designed as a 4 week balance training program. Outcome measures were Berg's balance scale, Multidirectional reach test and CTSIB. Balance was trained by making the subject perform balance exercises standing over a square foam surface which reduces the quality of surface orientation input. Training was given for 15-20 mins/day, 5 days in a week, for a period of 1 month. We observed a $25 \%$ increase in values of FFR, BFR, LFR \& RFR for multi directional reach test. Overall Berg's balance score improved from 48 to 54. CTSIB assessed before the training showed a poor performance in conditions $5 \& 6$, post training assessment showed an improvement of 12 seconds for condition 5 and 11 seconds for condition 6 . The results suggest that sensory-specific balance exercise has a positive training effect on balance among subjects with Parkinsonism.
\end{abstract}

Keywords: Parkinson's disease, Proprioceptive training, Balance exercises, CTSIB

\section{Introduction}

Parkinson's disease (PD) is a progressive neurologic disorder characterized by insidious onset. The first clinical sign occurs when about $60 \%$ of the dopamine-producing cells in the substantia nigra have degenerated (Booij J et al 1999). The mean age of onset of PD is in the mid fifties, with increasing incidence and prevalence as age increases (Ng DC 1996). The disease features impairment of resting muscle tone and voluntary movement, because of loss of striatal dopamine in the nigrostriatal dopamine pathway (Kish SJ, Shannnak K and Hornykiewcz O 1988). Clinical signs of bradykinesia, rhythmic tremor, rigidity, and postural instability follow dopamine depletion (Olanow CW and Koller WC 1998). Optimal management of Parkinson's disease (PD) involves both pharmacologic treatment and encouragement of physical activity according to American Academy of Neurology (1993) There is mounting evidence for the effects of physiotherapy in all stages of the disorder, yet few well-controlled prospective studies have documented the benefits of physical activity in PD (Comella CL et al 1994, Palmer SS et al 1986, Toole T et al 2000).

Physical therapists teach clients with PD, strategies to cope with impairments and disabilities, ideally allowing them to move easier, minimize disability, and retain independent living skills. Physical therapists also play a role in assessing the ability of people with PD to accomplish complex tasks, such as shopping, that are routinely performed in everyday life. Therapists are called upon to measure and assess changes in function, disability, activity, and response to therapy. In addition, therapists are often required to measure and assess changes in the disease, including medication changes and surgical interventions, as well as to monitor the natural progression of the disease (Canning CG et al 1997, Morris ME Iansek R Churchyard A 1998, Morris ME 2000, Schenkman M et al 1989, and Schenkman M Butler RB 1998). Postural instability has been noted to be one of the major causes for frequent falls among these subjects. Balance exercises have been frequently used among these subjects to 
prevent falls and work done by Mark A. Hirsch et.al (2000) has shown that the use of high-intensity resistance training and balance training can be safely used in improving balance and strength.

Balance is the ability to control the body mass or centre of mass or integrated sensory and musculoskeletal system and is modified within the CNS in response to changing internal and external environmental conditions The upright postures like sitting, standing or walking requires proper functioning of central balancing mechanisms. Afferent impulses of widespread origins, including signals from the periphery, play important role in eliciting and guiding responses, while efferent pathways carry messages to the muscles for the execution of the balancing act. Damage to any one of the central mechanisms or interruption anywhere along the sensory or motor pathway may lead to an inability to maintain body's COG within the base of support, but, no one system directly specifies the position of COG as stated by Brunnstrom (1970). Shumway Cook (1986) stated information from the vestibular system is a powerful source in maintaining postural control and it provides the CNS with information about the position and movement of the head with reference to gravity and inertial force providing gravito-inertial frame of reference for postural control. The visual inputs report information regarding the position and motion of head with respect to supporting surface. The somatosensory system provides CNS with position and motion information about body with reference to supporting surface and in addition provides information about the relationship of body segments to each other. Each of the senses provides the CNS with specific information about the relationship of body segments to each other and also a different frame of reference for postural control. The differing roles of sensory inputs is controversial but it is likely that they are co-ordinated in a task related manner which is dependent on environmental circumstances. Winter et al (1990) stated that the redundancy present within the sensory systems in the maintenance of balance enables not only the verification of inputs which may be conflicted but also allows for compensation. Work done by Richard D Di Fabio (1991) stated that disease alters one sensory modality then balance reaction can be compensated through the use of remaining sensory inputs.

Evidence (Stanley John Winser and Priya Stanley 2009) has shown that improving the influence of proprioceptors among clients with stroke improves balance. Studies done on stroke (Isabelle V. Bonan et al 2004) have administered balance exercises with deprived vision in order to reduce visual dependence in improving balance. Standing over a smooth / compliant surface can be used to facilitate the proprioceptive inputs. And we hypothesized that the improvement in the inputs from proprioceptors would improve balance. In the review done we found a paucity of literature on the influence of proprioceptors on balance among subjects suffering from Parkinson's disease. Thus this case study is intended to document the influence of training proprioceptor in improving balance among Parkinson's disease.

\section{Case report}

A 65 year old ambulant subject presented with complaints of imbalance, early fatigue, lack of interest in all social activities for the past 3 years. No history of infection/ illness or accidents in the past 5 years $\&$ he is on medication for Diabetes mellitus for 7 years. He was diagnosed to have idiopathic Parkinson's disease in a local hospital. Subject is a retired school teacher staying at home with his wife. He lives in the second floor of a 2 storied building, gets access to his home by climbing 2 flights of stairs having 24 steps. Currently he is independent in all activities except bathing and toileting for which he requires supervision from his wife.

Motor examination revealed good muscle efficiency in all 4 limbs, both precision and power of hand were good, all deep tendon reflexes (biceps, triceps, brachioradialis, quadriceps and tendo Achilles) were diminished. There was a mild non velocity dependent increase in the muscle tone which could be graded as 1+ in MAS. All sensory functions (peripheral and higher cortical) were normal. Co-ordination tests, including equilibrium and non equilibrium were negative which can be accounted for normal functioning of the cerebellum. The subject demonstrated an independent gait and the walking pattern showed mild features of festination. The walking speed was $69.5 \mathrm{mts} / \mathrm{min}$, with cadence of $128 / \mathrm{min}$, stride length of $128 \mathrm{cms}$ and step length $62 \mathrm{cms}$. Reciprocal arm swing while walking was preserved. FIM score of all ADL were 7except bathing and toileting which were grade 6. Based on the assessment the subject can be classified as stage 3 Parkinsonism in Hoehn and Yahr scale (Christopher G. Goetz et.al 2004).

\section{Material and methods}

A single case study of a 65 years old Parkinson's subject was considered. Approval from the Institute research board was obtained and the subject gave written consent to participate in the trial. The trial was designed as a 4 week balance training program. The inital assessment was done in May 2010 and the post training assessment was done after 30 days. 3 outcome measures were considered to assess the progress of patient's balance. 


\subsection{Outcome measures}

Outcome measures considered are Berg's balance scale, Multidirectional reach test and Clinical test for Sensory Integrity and Balance (CTSIB). Equipments used are shown in figure 1.

\subsubsection{Berg's balance scale (BBS)}

The BBS is an objective measure of balance abilities. It has been used to identify and evaluate balance impairment. The scale consists of 14 tasks common in everyday life. The items test the subject's ability to maintain positions or movements of increasing difficulty by diminishing the base of support from sitting and standing to single-leg stance. Subjects ability to change positions is assessed as well. Works done by Abu A. Qutubuddin et al concludes, BBS may be used as a screening and ongoing assessment tool for patients with PD.

\subsubsection{Multidirectional reach test}

This ingenious tool is a $250 \mathrm{~cm}$ 'inch' tape stuck to the wall. The tool measures forward functional reach (FFR), backward functional reach (BFR), and reach to the left and right of the subject to measure left functional reach (LFR) and right functional reach (RFR), respectively. The subject was positioned adjacent to wall with the shoulder at $0 \mathrm{~cm}$ of the inch tape. Shoulder was flexed to 90 degrees with elbow and fingers in full extension. The initial reading was taken from the tip of middle finger, then the subject was instructed to reach forward as far as possible without lifting any part of his foot or loosing balance and at the end point of reach the final reading was taken. Similarly backward reach was measured by instructing the patient to lean back. To assess right and left reach the subject was positioned by facing the wall and was instructed to reach to his left and then to his right. The subject's maximum reach was measured by calculating difference between the initial reach and the stretch reach. Assistance was taken to ensure safety.

\subsubsection{Clinical test for sensory integrity and balance (CTSIB)}

The CTSIB test was used to assess the contribution from sensory inputs (vision, vestibular, somatosensory) for maintaining balance. The test attempts to isolate the various sensory contributions, by either removing or distorting (via sway referring the visual surround or the surface platform) the visual, vestibular and/or somatosensory inputs needed for postural control. The resultant 6 conditions progress from the most stable (eye open, solid support surface) to the least stable (sway referenced vision and surface), as shown in figures 2 to 7 . Conditions are as follows:

Condition 1: Eye open + Firm surface

Condition 2: Eye closed + Firm surface

Condition 3: Visual conflict + Firm surface

Condition 4: Eye open + Compliant surface

Condition 5: Eye closed + Compliant surface

Condition 6: Visual conflict + Compliant surface

The support surface condition consists of a hard flat floor and square foam that reduces the quality of surface orientation input. The visual conflict dome was a custom made goggles with an opaque coving. By allowing the subject to open his eyes while using the opaque covered goggles during the test, the subject is provided with an altered visual reference. A stop watch was used to record the amount of time the subject maintained initial equilibrium position in each condition. Assistance was taken to ensure safety of the subject. Test interpretation: A poor score in conditions 1 and 2 indicated that the subject made poor use of somatosensory reference. A poor score in conditions 4 and 1 indicates, the subject uses poor visual reference. A poor score in conditions 5 and 1 indicated that the subject makes poor use of vestibular cues and a poor score in conditions 2, 3, 5 and 6 with inaccurate visual cues worse than no visual cue, indicates that the subject relies on visual cues even when they are inaccurate.

\subsection{Treatment protocol}

The subject's balance was trained by making him stand over a square foam surface which reduces the quality of surface orientation input and all activities related to balance were administered over the same mat. Training was given for 15-20 minutes/day, 5 days a week for a period of 1 month. The sequence of balance activity was kept constant for all treatment sessions. The sequence of activities are as follows: 2 to 3 minutes of warm ups for upper and lower limbs followed by repetition of the same set of activities with foot placed closer and then in tandem standing for 5 minutes. For the next 5 minutes the subject was asked to keep his eyes closed and repeat the same set of exercises. Help from an assistant was taken to ensure safety of the subject. The subject was given 
the freedom to open eyes when he felt excessive imbalance. Finally the last 3-5 minutes was utilized to train dynamic balance by asking the subject to catch ball and throw it back to the therapist as shown in figure 8 .

\section{Results}

Increment of score in all the 3 outcomes was observed. The multi directional reach test showed an overall increase in reach distance in all directions. We observed an average of $25 \%$ increase in values between pre and post training assessment. Berg's balance score of the patient was healthy during pre training assessment with an overall score of 48 and at the end of the trial the patient scored a maximum score of 54. CTSIB assessed before the training showed a poor performance in conditions $5 \& 6$ i.e the subject had difficulty in integrating proprioceptive inputs when the visual input is removed. Post training assessment showed an improvement of 12 seconds for condition 5 and 11 seconds for condition 6.

\section{Discussion}

The results of this experiment provide evidence that, the balance exercises dedicated to stimulate and facilitate the peripheral proprioceptors have beneficial effects in improving balance among individuals suffering from PD. Our investigation represent the first systematic evaluation of balance training by manipulating peripheral sensory inputs among the target population. Numerous findings emerged that were relevant to clinical practice. The most notable finding of our study were 1- Subjects with PD have difficulty in integrating peripheral sensory inputs especially the proprioceptive inputs which could be one of the contributing factors for impaired balance, 2- The contribution from the peripheral proprioceptors for maintaining equilibrium are trainable and 3- Balance training on a compliant surface can be used to facilitate proprioceptors to improve balance.

The results of the case study are interesting in several aspects. Firstly, the comparison of the results for the multidirectional reach test at the beginning and end of the training period showed increase in all direction. Newton RA, in his work had stated the muiltidirectional reach test to be an inexpensive, reliable, and valid tool that can be used to assess the stability of individuals. The results of this test reflects the increment in limits of stability in all directions. From the results it is apparent that the limits of stability has increased for all 4 domains. Secondly, we observed an increase in the overall score of the Berg's balance score. The BBS was designed to measure changes in functional standing balance over time. It is a 14-item scale that rates each function from 0 (worst) to 4 (best) along a dependence-independence continuum. This summative scale measures balance abilities seen during tasks involving sitting, standing, and positional changes. Total scores are indicative of overall balance abilities, with scores interpreted in the following manner: 0 to 20 , wheelchair bound; 21 to 40 , walking with assistance; and 41 to 56, independent (Berg KO et al 1998 and Berg K Wood-Dauphinee S Williams JI 1995). The subject was having an overall score of 48 before the trial with scores lacking in activities like Turning to look behind, Turning 360 degrees, placing alternate foot on stool, standing with one foot in front and standing on one foot. An inability to score maximum in these tasks could be due an inability to terminate the sequential activity. Meg E Morris states, these subjects have sustained discharge in the supplementary motor area (SMA), rather than the rapid drop in neural activity in the SMA that normally allows movements to be terminated. Difficulty terminating locomotor actions such as walking, running, or turning during walking is thought to be one of the major factors that predisposes people with PD to slips, trips, and falls (Morris ME and Iansek R 1997). The increase in the overall score following therapy incicates an improvement in the patients ability to terminate sequential activity effectively However we could not explain the reason for this phenomenon. Further studies are warranted to explore this phenomenon. Finally the CTSIB showed an improvement in conditions $5 \& 6$. Our previous studies done on stroke subjects revealed that this improvement could be because of unmasking of the somatosensory and vestibular systems in maintaining balance. Same explanation can be adopted to explain the phenomenon behind improvement in balance among the patients suffering from Parkinson's disease.

\section{Conclusion}

The results of this study suggested that sensory-specific balance exercise had a positive training effect on balance. However, 2 limitations should be mentioned. This was a pilot trial done on a single case, the results of which cannot be generalized therefore further studies can be carried out on more number of subjects. Secondly, blinding can be considered in future.

\section{References}

American Academy of Neurology. (1993). Assessment: posturography, Neurology, 43:1261-1264.

Abu A. Qutubuddin et al. (2005). Validating the Berg Balance Scale for Patients with Parkinson's Disease: A Key to Rehabilitation Evaluation: Arch Phys Med Rehabil, 86: 789- 792. 
Berg K, Wood-Dauphinee S, Williams JI. (1995). The Balance Scale: reliability assessment with elderly residents and patients with an acute stroke: Scand J Rehabil Med, 27:27-36.

Berg KO, Wood-Dauphinee SL, Williams JI, Maki B. (1992). Measuring balance in the elderly: validation of an instrument: Can J Public Health, 83(Suppl 2):S7-11.

Booij J, Tissingh G, Winogrodzka A, van Royen EA. (1999). Imaging of the dopaminergic neurotransmission system using single-proton emission tomography and positron emission tomography in patients with parkinsonism. Eur J Nucl Med, 26:171-82.

Brunnstrom. (1970). Walking preparation and gait training- trunk balance In: Brunnstrom's movement therapy in hemiplegia: a neurological approach; second edition: Medical Dept., Harper \& Row, 1970, Pg-146.

Canning CG, Alison JA, Allen NE, Groeller H. (1997). Parkinson's disease: an investigation of exercise capacity, respiratory function, and gait. Arch Phys Med Rehabil, 78:199-207.

Christopher G. Goetz et.al. (2004) Movement Disorder Society Task Force Report on the Hoehn and Yahr Staging Scale: Status and Recommendations. Movement Disorders, 19, No. 9, 1020-1028.

Comella CL, Stebbins GT, Brown-Toms N, Goetz CG. (1994). Physical therapy and Parkinson's disease: a controlled clinical trial. Neurology, 44: 376-378.

Isabelle V. Bonan et al. (2004). Reliance of visual information after stroke. Part II: Effectiveness of a balance rehabilitation program with visual cue deprivation after stroke: A randomized control trial. Arch Phy Med Rehabil, 85: 268-273.

Kish SJ, Shannnak K, Hornykiewcz O. (1988). Uneven pattern of dopamine loss in the striatum of patients with idiopathic Parkinson's disease. N Engl J Med, 318:376-80.

Mark A. Hirsch, PhD, et al. (2003). The Effects of Balance Training and High-Intensity Resistance Training on Persons With Idiopathic Parkinson's Disease. Arch Phys Med Rehabil, 84: 1109-1117.

Meg E Morris. (2000). Movement Disorders in People with Parkinson Disease: A Model for Physical Therapy. Physical Therapy, 80: 578- 597.

Morris ME, Iansek R. (1997). Gait disorders in Parkinson's disease: a framework for physical therapy practice. Neurology Report, 21:125-131.

Morris ME. (2000). Movement disorders in people with Parkinson's disease: a model for physical therapy. Phys Ther, 80:578-597.

Morris ME, Iansek R, Churchyard A. (1998). The role of the physiotherapist in quantifying movement fluctuations in Parkinson's disease. Aust J Physiother, 44: 105-114.

Newton RA. (2001). Validity of the multi-directional reach test: a practical measure for limits of stability in older adults. J Gerontol A Biol Sci Med Sci., 56(4): M248-252.

Ng DC. (1996). Parkinson's disease. Diagnosis and treatment. West J Med, 165:234-240.

Olanow CW, Koller WC. (1998). An algorithm (decision tree) for the management of Parkinson's disease. Neurology, 50 Suppl 3:S 1-57.

Palmer SS, Mortimer JA, Webster DD, Bistevins R. (1986). Exercise therapy for Parkinson's disease. Arch Phys Med Rehabil, 67:741-5.

Richard D Di Fabio. (1986). Assessing the influence of sensory interaction on balance: suggestion from the field. Phys Ther, 66: 1548-1550.

Roberta A. Newton. (2001). Validity of the Multi-Directional Reach Test. A Practical Measure for Limits of Stability in Older Adults. The Journals of Gerontology, Series A 56: 248-252.

Schenkman M, Donovan J, Tsubota J, et al. (1989). Management of individuals with Parkinson's disease: rationale and case studies. Phys Ther, 69:944 -955.

Schenkman M, Butler RB. (1989). A model for multisystem evaluation and treatment of individuals with Parkinson's disease. Phys Ther, 69: 932-943.

Stanley John Winser et al. (2009). Dominance of sensory inputs in maintaining balance among acute and subacute stroke patients. Indian Journal of Physiotherapy and Occupational Therapy, 3: 79-84.

Toole T, Hirsch MA, Forkink A, Lehman DA, Maitland CG. (2000). The effects of a balance and strength training program on Parkinsonism: a preliminary study. J Neurol Rehabil, 14:165-74. 
Winter, D.A., Patla, A.E and Frank, J.S. (1990). Assessment of balance control in Humans. Medical Progress through Technology, 16: 31-51.

Table 1.

\begin{tabular}{|l|l|l|}
\hline Outcomes & Pre test score & Post test score \\
\hline Multi directional reach & FFR- 16cms & FFR- 20cms \\
& BFR- 7cms & BFR- 8cms \\
& LFR- 18cms & LFR- 21cms \\
& RFR- 14cms & RFR- 19cms \\
\hline Berg's balance score (overall) & 48 & 54 \\
\hline CTSIB & Condition 1- 30 sec & Condition 1- 30 sec \\
& Condition 2- 30 sec & Condition 2- 30 sec \\
& Condition 3- 30 sec & Condition 3- 30 sec \\
& Condition 4- 30 sec & Condition 4- 30 sec \\
& Condition 5- 15 sec & Condition 5- 27 sec \\
& Condition 6- 19 sec & Condition 6- 30 sec \\
\hline
\end{tabular}

Shows the progress in outcomes pre and post training

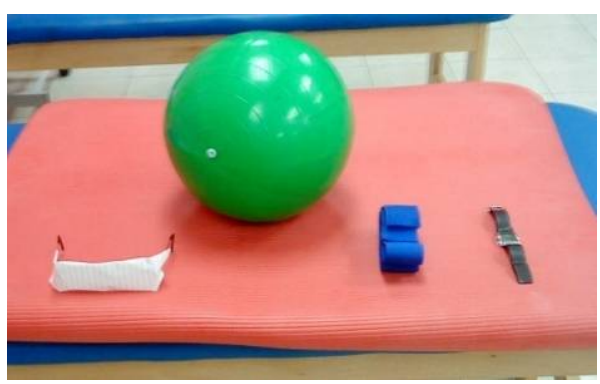

Figure 1. Equipments used (square red mat- compliant surface, opaque goggles- altered visual reference, ball to train balance, scarf to blind vision, stop watch)

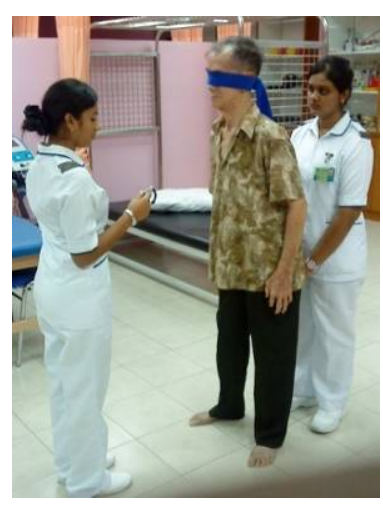

Figure 2. Condition 1of CTSIB (Eye open + Firm surface) 


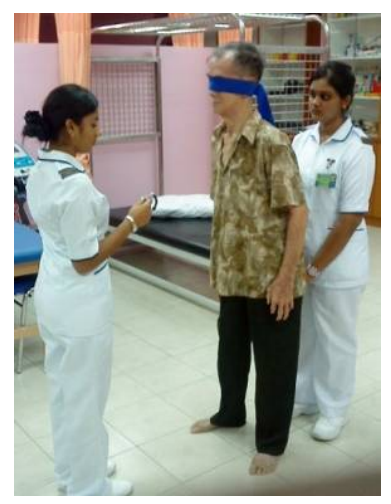

Figure 3. Condition 2 (Eye closed + Firm surface)

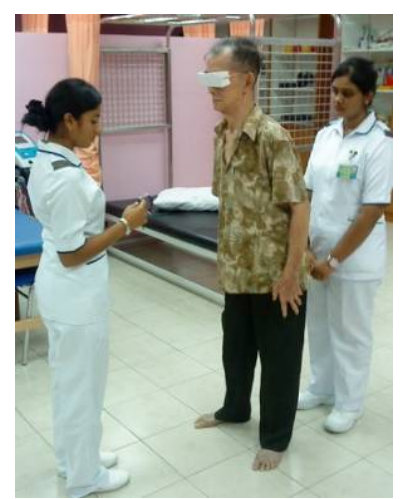

Figure 4. Condition 3 (altered visual reference + Firm surface)

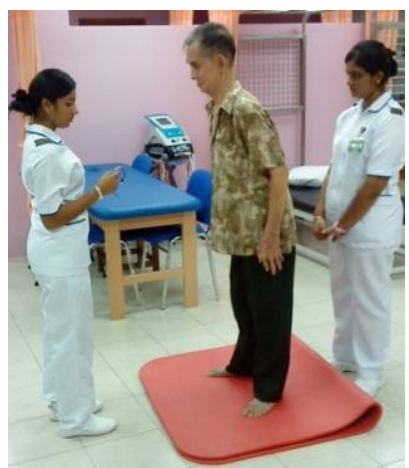

Figure 5. Condition 4 (eye open + compliant surface)

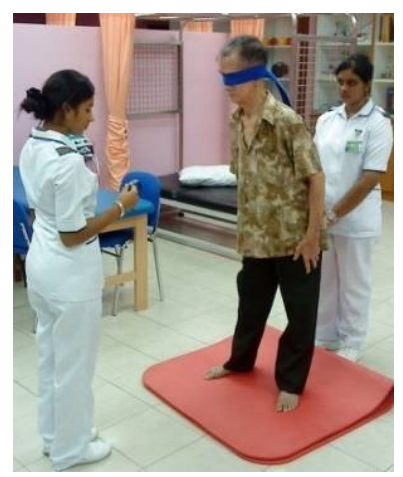

Figure 6. Condition 5 (eye closed + compliant surface) 


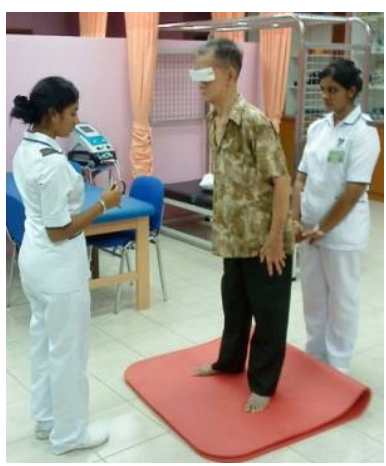

Figure 7. Condition 6 (altered visual reference + compliant surface)

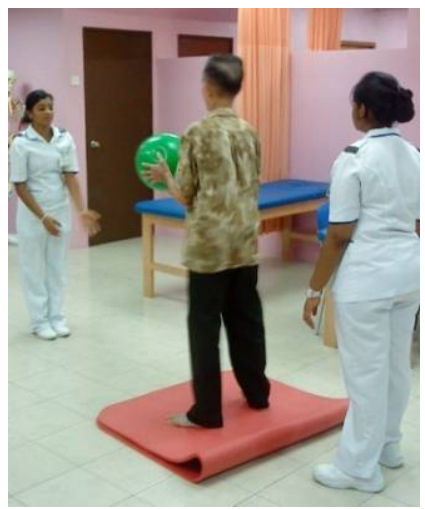

Figure 8. Subject undergoing conventional balance training standing over a compliant surface. 